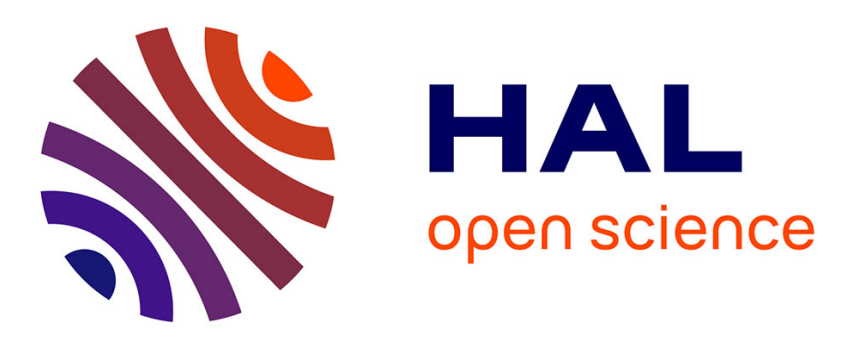

\title{
A Stochastic Collocation Method Combined With a Reduced Basis Method to Compute Uncertainties in Numerical Dosimetry
}

Amine Drissaoui, Stéphane Lanteri, Philippe Lévêque, François Musy, Laurent Nicolas, Ronan Perrussel, Damien Voyer

\section{To cite this version:}

Amine Drissaoui, Stéphane Lanteri, Philippe Lévêque, François Musy, Laurent Nicolas, et al.. A Stochastic Collocation Method Combined With a Reduced Basis Method to Compute Uncertainties in Numerical Dosimetry. IEEE Transactions on Magnetics, 2012, 48 (2), pp 563-566. 10.1109/TMAG.2011.2174347 . hal-00675034

\section{HAL Id: hal-00675034 \\ https://hal.science/hal-00675034}

Submitted on 2 Mar 2012

HAL is a multi-disciplinary open access archive for the deposit and dissemination of scientific research documents, whether they are published or not. The documents may come from teaching and research institutions in France or abroad, or from public or private research centers.
L'archive ouverte pluridisciplinaire HAL, est destinée au dépôt et à la diffusion de documents scientifiques de niveau recherche, publiés ou non, émanant des établissements d'enseignement et de recherche français ou étrangers, des laboratoires publics ou privés. 


\title{
A stochastic collocation method combined with a reduced basis method to compute uncertainties in numerical dosimetry
}

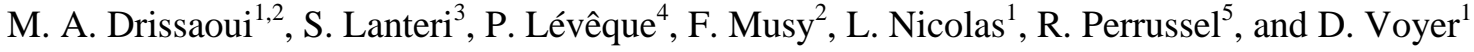 \\ ${ }^{1}$ Laboratoire Ampère (CNRS UMR 5005), CNRS-Université de Lyon, Ecole Centrale de Lyon, 69134 Ecully, France \\ ${ }^{2}$ Institut Camille Jordan (CNRS UMR 5208), CNRS-Université de Lyon, Ecole Centrale de Lyon, 69134 Ecully, France \\ ${ }^{3}$ INRIA, team NACHOS, 06902 Sophia Antipolis, France. \\ ${ }^{4}$ XLIM (UMR CNRS 6172), CNRS-Université de Limoges, 87060 Limoges, France. \\ ${ }^{5}$ LAPLACE (CNRS UMR 5213), CNRS-Université de Toulouse, INPT ENSEEIHT, 31071 Toulouse, France
}

\begin{abstract}
A reduced basis method is introduced to deal with a stochastic problem in a numerical dosimetry application in which the field solutions are computed using an iterative solver. More precisely, the computations already performed are used to build an initial guess for the iterative solver. It is shown that this approach significantly reduces the computational cost.
\end{abstract}

Index Terms - Finite-element methods, numerical analysis, dosimetry.

\section{INTRODUCTION}

$\mathrm{T}$ The lack of knowledge on the electric parameters of tissues raises an issue in computational electromagnetic applications such as numerical dosimetry [1]. The Stochastic Collocation Method (SCM) is an "attractive" technique to deal with this kind of problem because existing deterministic solvers can be readily applied like in the Monte Carlo sampling [2]. We use the SCM coupled with a Smolyak Adaptive Algorithm (SAA) [3] which enables to build the solution gradually by an adaptive choice for the realizations of the input random parameters (these realizations will be called the "collocation points"). Every step of the SAA involves several collocation points, and each of them requires a deterministic computation that can be numerically expensive in realistic applications. When the deterministic computations are performed sequentially using an iterative solver, the computations already performed could be used to reduce the computational cost. The authors of [4] proposed to choose as the initial guess for the iterative solver the previous computed solution for which the collocation point is "close" to the next considered point. Here, we propose to compute an initial guess with the Reduced Basis Method (RBM); this practical aspect is not studied in the RBM papers [5,6]. "Similar" ideas have already been used in a transient implicit time integration process [7] but their approach was not dedicated to systems with varying parameters. Moreover, we couple the RBM with an A Posteriori Error Indicator (APEI) to choose the next collocation point.

First we review some aspects of the RBM; for complementary details see also [5]. Then, numerical experiments on a wave equation problem show that the computational cost of the solver can be significantly reduced. Finally, we point out some limitations due to the nature of the SAA.

Manuscript received July 1, 2011. Corresponding author: A. Drissaoui. Author (e-mail: Amine.Drissaoui@ec-lyon.fr).

Digital Object Identifier inserted by IEEE

\section{REDUCED BASIS METHOD}

\section{A. Finite element approximation}

We are interested in solving the time-harmonic Maxwell equations in a 3D domain $\Omega=\bigcup_{\mathrm{k}=1}^{\mathrm{p}} \Omega_{\mathrm{k}}$, where the $\Omega_{\mathrm{k}}$ are nonoverlapping subdomains. Each subdomain $\Omega_{\mathrm{k}}$ is characterized by a constant electric parameter $\tilde{\varepsilon}^{\mathrm{k}}=\varepsilon_{0} \varepsilon_{\mathrm{r}}^{\mathrm{k}}+\sigma^{\mathrm{k}} / \mathrm{j} \omega$, where $\varepsilon_{0}$ denotes the vacuum permittivity, $\omega$ the angular frequency, $\varepsilon_{\mathrm{r}}^{\mathrm{k}}$ the relative permittivity and $\sigma^{\mathrm{k}}$ the conductivity of the subdomain $\Omega_{\mathrm{k}}$.

In the stochastic context, the parameters $\varepsilon_{\mathrm{r}}^{\mathrm{k}}$ and $\sigma^{\mathrm{k}}$ are considered as independent random variables. For a given realization of these random variables, the weak formulation to compute the electric field $\overrightarrow{\mathrm{E}}$ on a conforming Finite Element (FE) space $\mathrm{X}_{\mathrm{h}}$ can be written:

$$
\mathrm{a}\left(\overrightarrow{\mathrm{E}}(\widetilde{\varepsilon}), \overrightarrow{\mathrm{E}}^{\prime}, \tilde{\varepsilon}\right)=\mathrm{f}\left(\overrightarrow{\mathrm{E}}^{\prime}\right) \forall \overrightarrow{\mathrm{E}}^{\prime} \in \mathrm{X}_{\mathrm{h}}, \tilde{\varepsilon}=\left(\tilde{\varepsilon}^{1}, \ldots, \tilde{\varepsilon}^{\mathrm{p}}\right),
$$

$$
\text { with } \begin{aligned}
& \mathrm{a}\left(\overrightarrow{\mathrm{E}}, \overrightarrow{\mathrm{E}}^{\prime}, \tilde{\varepsilon}\right)=\mathrm{a}_{0}\left(\overrightarrow{\mathrm{E}}, \overrightarrow{\mathrm{E}}^{\prime}\right)-\sum_{\mathrm{k}=1}^{\mathrm{p}} \omega^{2} \mu_{0} \tilde{\varepsilon}^{\mathrm{k}} \mathrm{a}_{\mathrm{k}}\left(\overrightarrow{\mathrm{E}}, \overrightarrow{\mathrm{E}}^{\prime}\right), \\
& \mathrm{a}_{0}\left(\overrightarrow{\mathrm{E}}, \overrightarrow{\mathrm{E}}^{\prime}\right)=\int_{\Omega} \nabla \times \overrightarrow{\mathrm{E}} \cdot \overline{\nabla \times \overrightarrow{\mathrm{E}}^{\prime} \mathrm{dx}+\mathrm{BT},} \\
& \mathrm{a}_{\mathrm{k}}\left(\overrightarrow{\mathrm{E}}, \overrightarrow{\mathrm{E}}^{\prime}\right)=\int_{\Omega_{\mathrm{k}}} \overrightarrow{\mathrm{E}} \cdot \overline{\overrightarrow{\mathrm{E}}^{\prime}} \text { for } 1 \leq \mathrm{k} \leq \mathrm{p}, \\
& \text { and } \mathrm{f}\left(\overrightarrow{\mathrm{E}}^{\prime}\right)=-\mathrm{j} \omega \mu_{0} \int_{\Omega} \overrightarrow{\mathrm{J}}_{\mathrm{s}} \cdot \overline{\overrightarrow{\mathrm{E}}^{\prime}} .
\end{aligned}
$$

$\nabla \times$ denotes the curl operator, $\mu_{0}$ the vacuum permeability, $\overrightarrow{\mathrm{J}}_{\mathrm{S}}$ the electric current source, and BT the boundary term.

The solution of (1) leads to a large sparse linear system:

$$
\left(\mathrm{A}_{0}-\omega^{2} \mu_{0} \sum_{\mathrm{k}=1}^{\mathrm{p}} \widetilde{\varepsilon}^{\mathrm{k}} \mathrm{A}_{\mathrm{k}}\right) \mathrm{E}(\widetilde{\varepsilon})=\mathrm{F},
$$

where $A_{k}$ and $F$ denote the matrix representation of the sesquilinear forms $\mathrm{a}_{\mathrm{k}}$ and semilinear form $\mathrm{f}$ in the standard basis of $X_{h}$, denoted here by $\left\{\vec{E}_{i}^{\prime} ; i=1\right.$ to $\left.n\right\}$.

Since they do not depend on $\tilde{\varepsilon}$, we can pre-assemble in an offline procedure the corresponding values:

$A_{k_{i}, j}=a_{k}\left(\vec{E}_{j}^{\prime}, \vec{E}_{i}^{\prime}\right)$ for $i, j=1$ to $n$ and $k=0$ to $p$,

$F_{i}=f\left(\vec{E}_{i}^{\prime}\right)$ for $i=1$ to $n$.

Thus the matrices $\left(A_{k}\right)_{0 \leq k \leq p}$ and $F$ do not require any calculation when we change the values of $\tilde{\varepsilon}$. 
In part III, a 3D dosimetry problem with a human head that involves several million unknowns is studied. Consequently the solution of the linear system (2) is computed using an iterative solver. In order to make a smart choice of its initial guess, we propose to build a Reduced Basis from the computed solutions obtained with the previous values of $\tilde{\varepsilon}$.

\section{B. Reduced basis approximation}

Suppose that the problem (1) has been solved for M different values of the parameters $\tilde{\varepsilon}$. In the reduced basis formulation, the local FE space $X_{h}$ in problem (1) is substituted by a space of functions $\mathrm{X}_{\mathrm{b}} \subset \mathrm{X}_{\mathrm{h}}$ spanned by the $\mathrm{M}$ known solutions. The reduced basis formulation is then:

$$
\mathrm{a}\left(\overrightarrow{\mathrm{E}}_{\mathrm{b}}(\widetilde{\varepsilon}), \overrightarrow{\mathrm{E}}^{\prime}, \tilde{\varepsilon}\right)=\mathrm{f}\left(\overrightarrow{\mathrm{E}}^{\prime}\right) \quad \forall \overrightarrow{\mathrm{E}}^{\prime} \in \mathrm{X}_{\mathrm{b}}
$$

For the purpose of well-conditioned systems, a modified Gram-Schmidt process depending on the tolerance $\alpha$ for the residual norm in the iterative solver is used to obtain an orthonormal basis $\left\{\vec{\xi}_{i} ; i=1\right.$ to $\left.N\right\}$ of $X_{b}$ with $N \leq M$. Usually, the space $X_{b}$ has a much smaller dimension than the dimension of $X_{h}(N<<n)$.

Then, the solution of (3) leads to a linear system

$$
\left(\mathrm{B}_{0}-\omega^{2} \mu_{0} \sum_{\mathrm{k}=1}^{\mathrm{p}} \tilde{\varepsilon}^{\mathrm{k}} \mathrm{B}_{\mathrm{k}}\right) \mathrm{E}_{\mathrm{b}}(\tilde{\varepsilon})=\mathrm{F}_{\mathrm{b}},
$$

where $B_{k}$ and $F_{b}$ denote the matrix representation of the sesquilinear forms $a_{k}$ and semilinear form $f$ in the basis $\left\{\vec{\xi}_{i} ; \mathrm{i}=1\right.$ to $\left.\mathrm{N}\right\}$. This "small" system is solved by Gaussian elimination.

In order to reduce the number of iterations for solving problem (1), we choose as the initial guess the solution to problem (3). Its expression in $X_{h}$ is given by the vector $\mathrm{WE}_{\mathrm{b}}(\tilde{\varepsilon})$ where $\mathrm{E}_{\mathrm{b}}(\tilde{\varepsilon})$ is the solution to the linear system (4) and $\mathrm{W}$ is the matrix $(\mathrm{n}, \mathrm{N})$ whose column $\mathrm{W}^{\mathrm{j}}$ contains the components of $\vec{\xi}_{j} \in X_{b}$ in the FE basis $\left\{\vec{E}_{i}^{\prime} ; i=1\right.$ to $\left.n\right\}$.

It is advantageous in terms of computational cost to calculate the matrices $B_{k}$ and the vector $F_{b}$ by using the relations $B_{k}=\bar{W}^{t} A_{k} W, k=0$ to $p, F_{b}=\bar{W}^{t} F$, where $\bar{W}^{t}$ is the conjugate transpose of $W$. Each addition of an element in the reduced basis only requires the calculation of one new line and one new column for $B_{k}$ and one new scalar for $F_{b}$. Note that once the matrices $B_{k}, k=0$ to $p$ and the vector $F_{b}$ are computed, the cost to build the matrix $\mathrm{B}_{0}-\omega^{2} \mu_{0} \sum_{\mathrm{k}=1}^{\mathrm{p}} \tilde{\varepsilon}^{\mathrm{k}} \mathrm{B}_{\mathrm{k}}$ and to solve the linear system (4) becomes independent of $n$ (it only depends on $\mathrm{p}$ and $\mathrm{N}$ ).

\section{A posteriori error indicator APEI}

Every step of the SAA involves a set of collocation points $\Sigma$ that have to be calculated. An APEI is introduced to order the set $\Sigma$ as usually done in the RBM [6]. Problem (4) is first solved for the different values of $\tilde{\varepsilon}$ corresponding to the collocation points belonging to $\Sigma$. An error indicator is then defined by using the residual norm of the linear system (2):

$$
\eta(\widetilde{\varepsilon})=\left\|\mathrm{A}(\widetilde{\varepsilon}) \mathrm{WE}_{\mathrm{b}}(\widetilde{\varepsilon})-\mathrm{F}\right\|^{2} \quad \forall \widetilde{\varepsilon} \in \Sigma,
$$

with $\mathrm{A}(\widetilde{\varepsilon})=\mathrm{A}_{0}-\omega^{2} \mu_{0} \sum_{\mathrm{k}=1}^{\mathrm{p}} \tilde{\varepsilon}^{\mathrm{k}} \mathrm{A}_{\mathrm{k}}$, and $\|$.$\| is the euclidian$ norm. In a similar way to the construction of (4), we precompute in an offline procedure the quantities related to this residual:

$$
\begin{aligned}
& {\overline{\left(A_{k} W^{i}\right)}}^{t} A_{k^{\prime}} W^{j}, \quad k, k^{\prime}=1 \text { to } p, \quad i, j=1 \text { to } N \text {, } \\
& \left(\mathrm{A}_{\mathrm{k}} \mathrm{W}^{\mathrm{i}}\right) \mathrm{F}, \quad \mathrm{k}=1 \text { to } \mathrm{p}, \quad \mathrm{i}=1 \text { to } \mathrm{N} \text {. }
\end{aligned}
$$

Then, we choose $\widetilde{\varepsilon}_{\mathrm{m}}=\operatorname{argmax}_{\tilde{\varepsilon} \in \Sigma}(\eta(\widetilde{\varepsilon}))$ as the next collocation point for which system (2) has to be solved. This approach is described in Algorithm I for 1 steps of the SAA.

\section{ALGORITHM I}

CONSTRUCTION OF THE REDUCED BASIS AND USE OF THE APEI

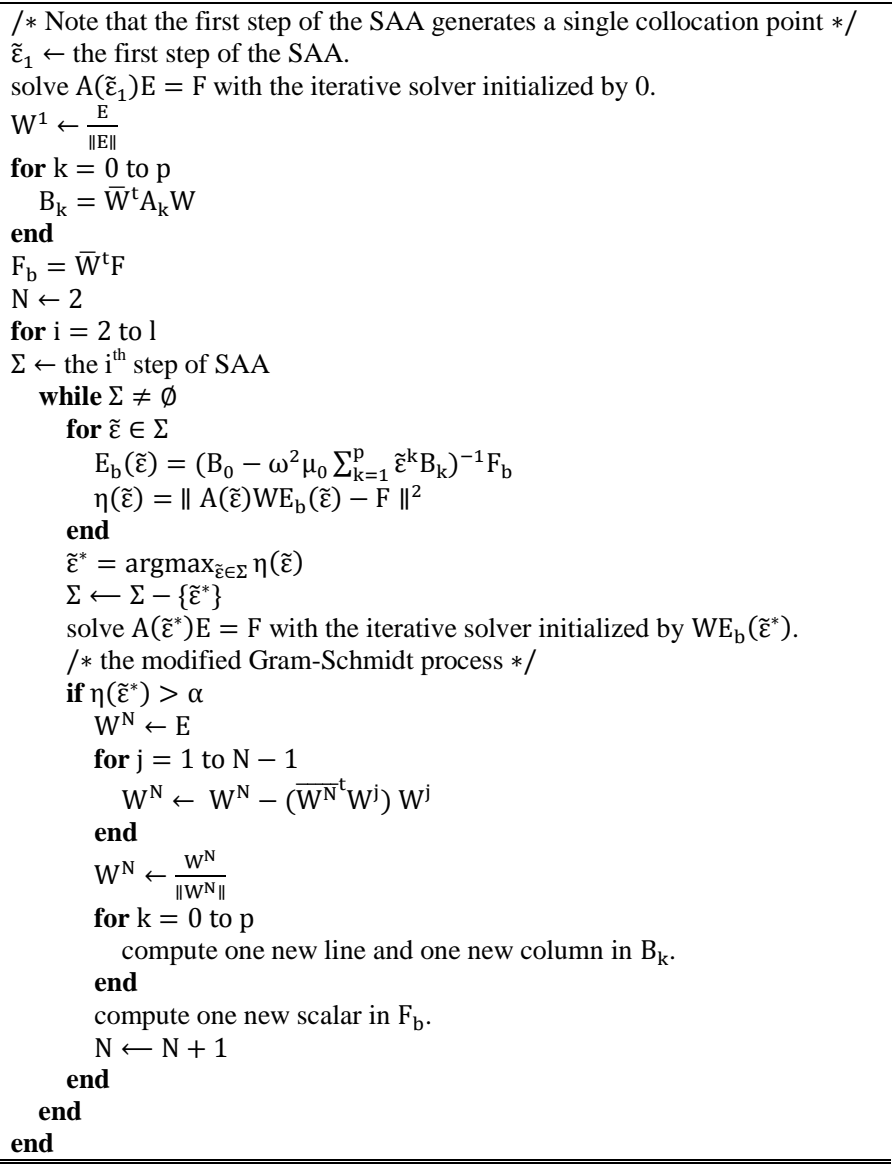

\section{RBM cost}

The computational cost is evaluated by the number of Arithmetic Operations (AO). Considering a Reduced Basis of dimension $\mathrm{N}$, the computational cost to obtain the initial guess $\mathrm{WE}_{\mathrm{b}}(\widetilde{\varepsilon})$ concerns the building of the linear system (4) that depends in one hand on the previous solutions expressed in the $\mathrm{FE}$ basis of dimension $\mathrm{n}$ and in the other hand on the solving of this system of dimension $\mathrm{N} \times \mathrm{N}$. Without using the APEI, the cost $\mathrm{C}_{\mathrm{RBM}}$ can be estimated as follows:

$$
\mathrm{C}_{\mathrm{RBM}} \leq \mathrm{pN}^{4}-2 \mathrm{pN}^{3}+9 \mathrm{nN}^{2}+6 \mathrm{CnN}+4 \mathrm{n},
$$

where $C$ is the maximum non-zero elements in any line of the matrices $\left(A_{k}\right)_{0 \leq k \leq p}$.

Using the APEI, the computational cost to determine $\tilde{\varepsilon}_{\mathrm{m}}$ grows with the evaluation of the residual (5) for all collocation points belonging to $\Sigma$. Considering a set $\Sigma$ of $\mathrm{N}$ points, the cost $\mathrm{C}_{\mathrm{APEI}}$ can be estimated as follows:

$$
\begin{aligned}
\mathrm{C}_{\mathrm{APEI}} \leq & (\mathrm{p}+1)^{2} \mathrm{~N}^{4}+\left(2 \mathrm{p}^{2}+5 \mathrm{p}+3\right) \mathrm{N}^{3}+ \\
& \left(2 \mathrm{n}+2(\mathrm{p}+2)^{2}\right) \mathrm{N}^{2}+ \\
& \left(2 \mathrm{n}+(\mathrm{p}+2)^{2}\right) \mathrm{N}+2 \mathrm{n} .
\end{aligned}
$$


Furthermore, the cost in solving (2) with the iterative solver initialized by 0 depends on the number of iterations made by the iterative solver. One have to keep in mind that one iteration to solve a problem of dimension $\mathrm{n}$ costs $2 \mathrm{Tn}[8,9]$, where $\mathrm{T}$ is constant that depends mostly on the preconditioning. In the application proposed in part III, we focus our attention in the number of iterations saved by the RBM with and without the APEI.

\section{APPLICATION TO A DOSIMETRY PROBLEM}

The dosimetry problem studied in this paper concerns the exposition of the Visible Human head to the electromagnetic radiation of a mobile phone as described in [10]. The mobile phone is formed by a monopole antenna and a chassis embedded in plastic, with the excitation point at the base of the antenna. The model of the Visible Human head is constituted of four biological tissues: the brain, the cerebro spinal fluid, the skull and the skin.

As introduced in Section II-A, the problem is solved using the FE method applied to the time-harmonic Maxwell equation. The mesh for the problem considered here is illustrated in Fig. 1. Using the lowest order edge elements in the discretization, the numerical problem involves 1.9 million unknowns. For this problem, the solution of (2) is computed using the Conjugate Orthogonal Conjugate Gradient (COCG) [8] with a potential projection preconditioning technique [9]. The COCG is stopped when the residual norm converges to the desired tolerance $\alpha=10^{-6}$. We do not focus our attention to evaluate the total numerical error (the error owing the numerical model and the iterative solver).

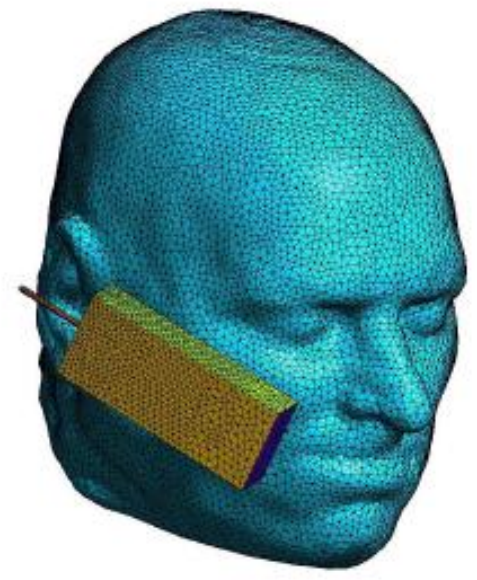

Fig. 1. Mesh of the Visible Human head with the mobile phone.

The quantity of interest in electromagnetic dosimetry is the SAR that measures the quantity of electromagnetic power absorbed by $1 \mathrm{~kg}$ of tissue:

$$
\mathrm{SAR}=\frac{1}{2} \frac{\sigma|\mathrm{E}|^{2}}{\rho}(\mathrm{W} / \mathrm{Kg})
$$

where $|E|$ is the amplitude of the electric field and $\rho$ the volumic mass in the considered tissue.

We consider that the relative permittivity and conductivity of the 4 tissues are random variables with uniform laws:

$\varepsilon_{\mathrm{r}}^{\mathrm{i}} \sim \mathrm{U}\left(\varepsilon_{\mathrm{r}_{\text {min }}}^{\mathrm{i}}, \varepsilon_{\mathrm{r}_{\text {max }}}^{\mathrm{i}}\right)$, and $\sigma^{\mathrm{i}} \sim \mathrm{U}\left(\sigma_{\text {min }}^{\mathrm{i}}, \sigma_{\text {max }}^{\mathrm{i}}\right) 1 \leq \mathrm{i} \leq 4$.
More precisely, uniform laws have been chosen with a mean value equal to the value referred in the well-known Gabriel database [11] and with a range of variation of $\pm 10 \%$ around the mean value.

To test the efficiency of the RBM, 4 strategies to choose the initial guess in the COCG are tested:

(i) the zero vector,

(ii) the nearest previous solution following a distance which is defined in function of the input parameters as in [4]:

$$
\mathrm{d}\left(\tilde{\varepsilon}_{\mathrm{s}}, \tilde{\varepsilon}_{\mathrm{t}}\right)=\sum_{\mathrm{i}=1}^{4} \frac{\left(\varepsilon_{\mathrm{r}_{\mathrm{s}}}^{\mathrm{i}}-\varepsilon_{\mathrm{r}_{\mathrm{t}}}^{\mathrm{i}}\right)^{2}}{\left(\varepsilon_{\mathrm{r}_{\text {max }}}^{\mathrm{i}}-\varepsilon_{\mathrm{r}_{\text {min }}}^{\mathrm{i}}\right)^{2}}+\sum_{\mathrm{i}=1}^{4} \frac{\left(\sigma_{\mathrm{s}}^{\mathrm{i}}-\sigma_{\mathrm{t}}^{\mathrm{i}}\right)^{2}}{\left(\sigma_{\text {max }}^{\mathrm{i}}-\sigma_{\text {min }}^{\mathrm{i}}\right)^{2}},
$$

where $\tilde{\varepsilon}_{s}, \tilde{\varepsilon}_{t}$ are two realizations of $\tilde{\varepsilon}, \square_{\min }$ and $\square_{\max }$ respectively denote the minimum and maximum values of the variable $\square$.

(iii) the reduced basis approximation without using APEI,

(iv) the reduced basis approximation by using APEI.

Strategy (ii) is based on the search of the minimum distance for the input parameters of the previous solution in order to choose the previous solution that will be considered as the initial guess for the current computation. In strategy (iv), a residual is computed and it gives an indicator of the error (it can be seen as a distance) between the initial guess built with the RBM and the exact solution. This last strategy is on the contrary based on the search of the maximum of this distance in order to improve the efficiency of the RBM.

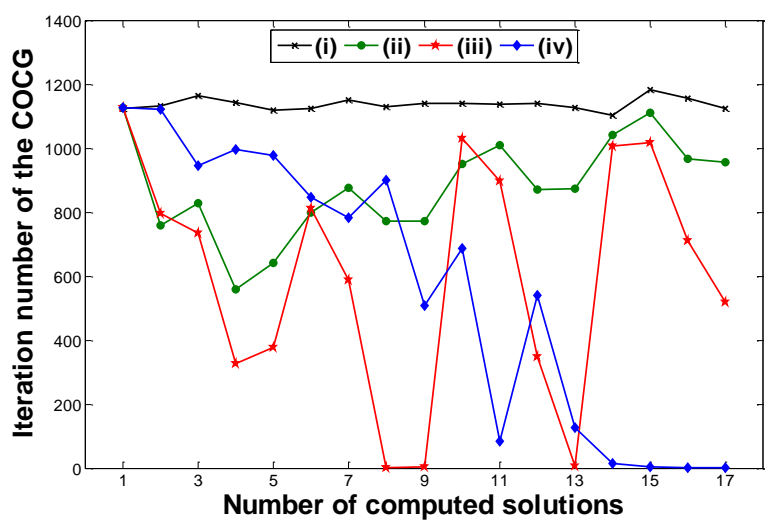

Fig. 2. Iteration number of the solver vs. the number of computed solutions

The efficiency of each approach is evaluated by a convergence study of the COCG in computing the 17 collocation points generated by the two first steps of the SAA (two step of SAA are enough to reach an error of $10^{-2}$ on the variance of the SAR). Results are reported in Fig. 2: the strategies using the RBM approximation need less iterations than (i) and (ii). The total number of the iterations to solve the 17 linear systems (2) is: (i) 19333, (ii) 14912, (iii) 10304, and (iv) 9665 iterations.

Strategies (iii) and (iv) seem to be the most attractive strategies since they save the more iterations. However one has to take into account the computational cost to build the RBM and the APEI. Based on the evaluated costs given in part II-D, Table I synthesizes the real cost of each strategy. It appears that strategies (iii) and (iv) remain more attractive than strategy (ii). Strategy (iv) save more computational cost compared to strategy (iii). 
TABLE I

COMPARISON BETWEEN THE COST SAVED IN THE DIFFERENT STRATEGIES

\begin{tabular}{|c|c|c|c|}
\hline Strategies & (ii) & (iii) & (iv) \\
\hline $\begin{array}{l}\text { Cost using formulas (6) and (7) with } \\
\qquad \begin{array}{c}\mathrm{C}=19, \mathrm{p}=4, \mathrm{n}=1.9 \mathrm{e}+6 \text { and } \\
\mathrm{N}=16(\mathrm{AO})\end{array}\end{array}$ & small & $7.8 \mathrm{e}+9$ & $8.9 \mathrm{e}+9$ \\
\hline $\begin{array}{c}\text { Number of iterations using the } \\
\text { solver }\end{array}$ & 14912 & 10304 & 9665 \\
\hline $\begin{array}{l}\text { Number of iterations saved } \\
\text { compared to strategy (i) }\end{array}$ & 4421 & 9029 & 9668 \\
\hline $\begin{array}{c}\text { Cost for the iterations saved with } \\
\qquad \mathrm{T}=1(\mathrm{AO})\end{array}$ & $1.7 \mathrm{e}+10$ & $3.4 \mathrm{e}+10$ & $3.7 \mathrm{e}+10$ \\
\hline Cost saved $=$ line $4-$ line $1(\mathrm{AO})$ & $1.7 \mathrm{e}+10$ & $2.6 \mathrm{e}+10$ & $2.8 \mathrm{e}+10$ \\
\hline
\end{tabular}

In agreement with the construction of the APEI, Fig. 3 shows that the value of the maximum of the APEI decreases monotically, and that its trend is nearly the same for the number of iterations in strategy (iv).

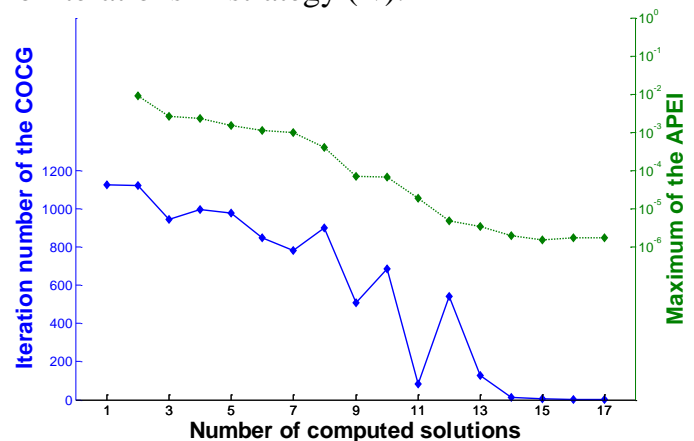

Fig. 3. Evolution study of $\max _{\tilde{\varepsilon} \in \Sigma}(\eta(\tilde{\varepsilon}))$

The interest of strategy (iv) appears much more if one goes further in the SAA. Fig. 4 gives the results for ten steps of the SAA (it gives an error of $10^{-6}$ on the variance of the SAR). The total number of the iterations to solve the 161 linear systems (2) is: (iii) 24637 , and (iv) 22916 iterations. But in this case, Fig. 5 shows that the APEI does not decrease monotically: at each step of SAA it appears an increase of the residual indicator. Actually, the APEI is in conflict with the variance indicator of the SAA [3].

In a last numerical experience called strategy (v), we apply Algorithm I where 1 is equal to 2 and $\Sigma$ contains the 160 collocation points obtained from strategy (iii) after ten steps of the SAA. It is fictional but will highlight the interest of the APEI. The total number of iterations to solve the 161 linear systems (2) is: 22205 iterations. The value of the maximum of the APEI decreases monotically on the 160 points (see Fig. 4 and 5).

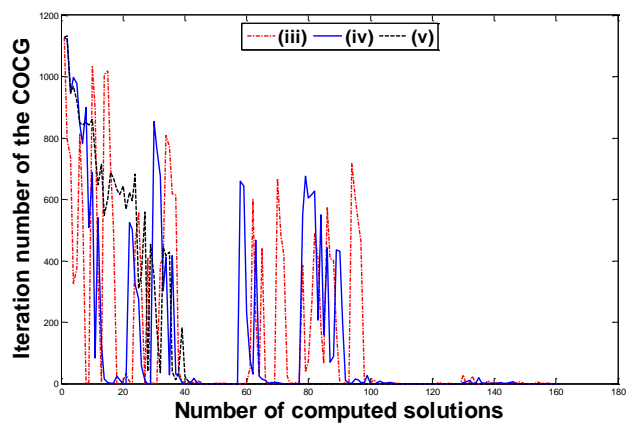

Fig. 4. Iteration number of the solver vs. the number of computed solutions

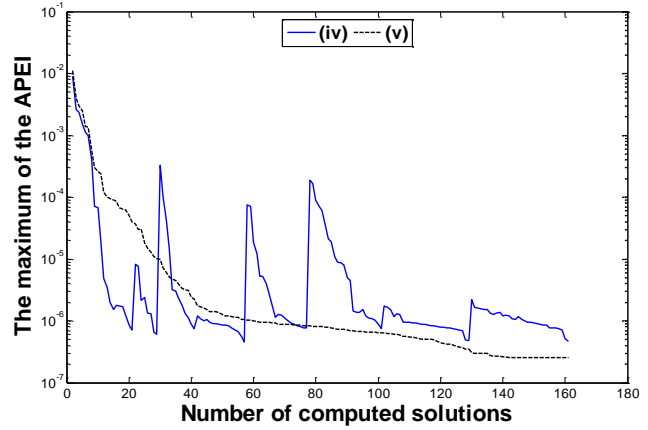

Fig. 5. Evolution study of $\max _{\tilde{\varepsilon} \in \Sigma}(\eta(\tilde{\varepsilon}))$

\section{CONCLUSION}

A stochastic collocation method combined with a reduced basis method has been proposed to study the variability in a 3D dosimetry problem. In the usual stochastic approach, the deterministic calculations are performed separately at the different collocation points without using previous computations. In order to save time in solving the different deterministic problems, it has been proposed to use a RBM without losing the accuracy. This method is efficient because it reduces the number of iterations of the iterative solver; however the adaptivity of the SAA seems in conflict with the APEI. Therefore, it is intended to replace the SAA by a statistical method like the Latin hypercube sampling.

\section{ACKNOWLEDGMENT}

This work was supported by the fondation Santé et Radiofréquences through the project entitled dosimétrie numérique statistique.

\section{REFERENCES}

[1] A. Hadjem, et al., "Study of Specific Absorption Rate (SAR) Induced in Two Child Head Models and in Adult Heads Using Mobile Phones," IEEE Transactions Microwave Theory and Techniques, vol. 53, no. 1,pp. 4-11, 2005.

[2] D. Xiu, "Fast Numerical Methods for Stochastic Computations: A Review," Communications In Computational Physics, vol. 5, no. 2, pp. 242-272, 2009

[3] D. Voyer, L. Nicolas, R. Perrussel, and F. Musy, "Comparison of methods for modeling uncertainties in a $2 \mathrm{~d}$ hyperthermia problem," Progress In Electromagnetics Research B, vol. 11, pp. 189-204, 2009.

[4] H. Zhu, et al., "A sparse grid based spectral stochastic collocation method for variations-aware capacitance extraction of interconnects under nanometer process technology," Proceedings of the conference on Design, automation and test in Europe, pp. 1514-1519, 2007.

[5] G. Rozza, D.B.P. Huynh, and A.T. Patera, "Reduced basis approximation and a posteriori error estimation for affinely parametrized elliptic coercive partial differential equations," Arch. Comput. Methods Eng., vol. 15, no. 3, pp. 229-275, 2008.

[6] M. Fares, J.S. Hesthaven, Y. Maday, and B. Stamm, "The reduced basis method for the electric field integral equation," Journal of Computational Physics, vol. 230, no. 14, pp. 5532-5555, 2011.

[7] M. Clemens, M. Wilke, R. Schuhmann, T. Weiland, "Subspace Projection Extrapolation Scheme for Transient Field Simulations," IEEE Transactions on Magnetics, vol. 40, no. 2, pp. 934-937, 2004.

[8] H. A. Van der Vorst, and J. B. M. Melissen, "A Petrov-Galerkin type method for solving $A x=b$, where $A$ is symmetric complex," IEEE Transactions on Magnetics, vol. 26, no. 2, pp. 706-708, 1990.

[9] R. Perrussel, L. Nicolas, and F. Musy, "An efficient preconditioner for linear systems issued from the finite-element method for scattering problems," IEEE Transactions on Magnetics, vol. 40, no. 2, pp. 10801083, 2004. 
[10] B. B. Beard et al., "Comparisons of Computed Mobile Phone Induced SAR in the SAM Phantom to That in Anatomically Correct Models of the Human Head," IEEE Transactions on Electromagnetic Compatibility, vol. 48, no. 2, pp. 397-407, 2006.

[11] C. Gabriel, S. Gabriel, and E. Corthout, "The dielectric properties of biological tissues: I. literature survey," Phys. Med. Biol., vol. 41, pp. 2231-2249, 1996. 